\title{
Improved Method for Disparity Estimation and Compression of Stereo Images with Varied Angle of Separation
}

\author{
D. Chandradevi \\ Research Scholar, \\ Dept. of Information Technology \\ Bharathiar University, Coimbatore \\ India
}

\author{
M. Sundaresan, PhD \\ Professor and Head, \\ Dept. of Information Technology, \\ Bharathiar University, Coimbatore \\ India
}

\begin{abstract}
This paper presents different sight point images of entity used to stereo image compression. The images are actual time images, stereo images of five objects taken between 10, 30 and 50 degree angles. The left image is used as reference image first and then right image has been used as the reference image. Images with different angles were intensified and outcomes were evaluated. In this work, compression time, decompression time and disparity results are superior when right image is used as the reference when compared to left image. But the PSNR values have shown a lower value that resulted in lesser quality of images.
\end{abstract}

\section{Keywords}

Stereo image compression, Disparity, Block matching algorithm

\section{INTRODUCTION}

\subsection{Digital Image Processing}

Digital images are electronic snapshots taken of a scene or scanned from documents such as photographs, manuscripts, printed texts and artwork. The digital image is composed of pixels arranged in a rectangular array with a certain height and width. Each pixel may consists of one or more bits of information representing the brightness of the image at that point and possibly including color information encoded as RGB triples. Images are usually taken from the real world via a digital camera, frame, or scanner or they may be generated by computer [1].

\subsection{Digital Image Compression}

Image compression is reducing the size of the file. As described in the fig. 1 Compression is the process of converting an input stream into another data stream that has a smaller size [2].

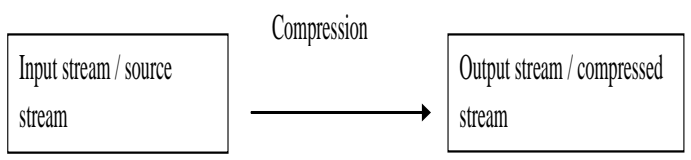

Fig 1: Process of compression

\subsection{Stereoscopic Images}

A stereoscopic picture consist of two views, one for each eye, and gives the viewer an illusion of three dimensional when seen with a suitable instrument, assuming that the viewer has two functioning eyes. Remote sensing, Photogrammetry, Robot visions, Computed Tomography, Magnetic Resonance
Imaging, Microsurgery, Space exploration, Conferencing, Virtual reality are the applications where using stereo images.

\subsection{Stereoscopic Image Compression}

Stereo image compression method, first compresses the reference image (left image) only, after that the block matching algorithm is applied between left and right image to identify the disparity values and then, they are encoded separately rather than compressing the whole right image [3].

\section{REVIEW OF LITERATURE}

T.Ramaprabha et al.[5] presented analysis of Stereo Image pair Compression non immersive virtual world reality using DCT II and BTC. Image compression may be lossy or lossless. Lossless compression is preferred for archival purposes and often for medical imaging, technical drawings, clip art, or comics. This is because lossy compression methods, especially when used at low bit rates, introduce compression artifacts. BTC is a recent technique used for compression of monochrome image data. It is one-bit adaptive moment-preserving quantize that preserves certain statistical moments of small blocks of the input image in the quantized output. The compressed image size in BTC (Block Truncation Coding) method is less than DCT II Compressed image size , the PSNR Value is high its image quality may be low after image is reconstructed. From the experimental result, the DCT II Image compression method provide high compression and efficient for stereo image pair used in Non Immersive Virtual Reality.

Tamas Frajka et al. [6] presented Residual Image Coding for Stereo Image Compression. One main focus of research in stereo image coding has been disparity estimation, a technique used to reduce the coding rate by taking advantage of the redundancy in a stereo image pair. Significantly less effort has been put into the coding of the residual image. These images display characteristics that are different from that of natural images. For this, proposed a new method for the coding of residual images that takes into account the properties of residual images. Particular attention is paid to the effects of occlusion and the correlation properties of residual images that result from block-based disparity estimation. The paper demonstrates that it is possible to achieve good results with a computationally simple method. And it focused on the coding of the residual image in a stereo image compression scenario. This method specifically addresses the issue of the image transform and the handling of the occluded blocks in the residual image. 


\section{METHODOLOGY}

In this proposed work, left image and after that right image is used as reference image and results are computed and compared. Stereo image pairs were taken in different angles of 30 and 40 degree. Testing and compression are done for each stereo image pairs.

\section{Stereo Image Encoding (Left Image as reference} image)

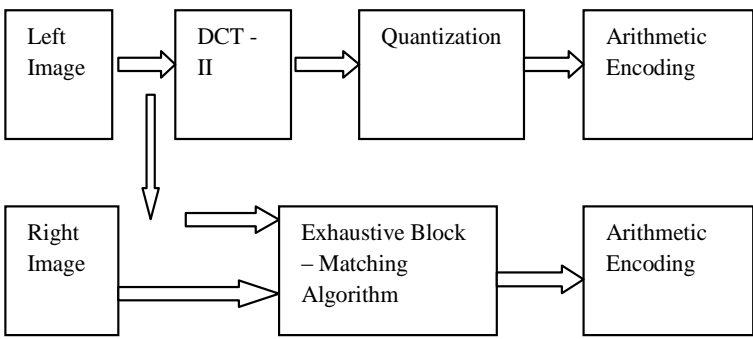

Fig 2: Block diagram for compressing a pair of Stereo Images by using Left image as reference image

Fig.2 explains the encoding process for left image used as reference image. And Fig.3 shows the encoding process for right image used as reference image.

\section{Stereo Image Encoding (Right Image as reference} image)

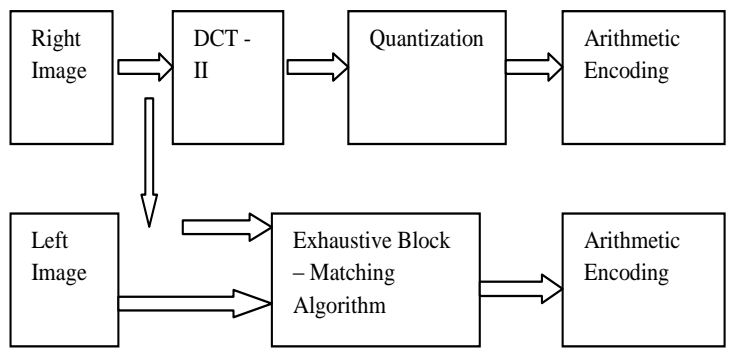

Fig 3: Block diagram for compressing a pair of Stereo Images by using Right image as reference image

\subsection{Preprocessing}

In this, all the images are taken in the .jpg format. PreProcessing is used for Noise Removal purpose and it take out good quality of images.

\subsection{Stereo Image Compression Using DCT II Transformation}

To remove the duplicate information of the neighboring pixels from the images, in this 2D Discrete Cosine Transform Technique used due to its strong energy compaction property [4]. In Stereo Image Pair compression using DCT II transform algorithm, the main objective is to decrease insignificance and redundancy of the image data and to store and transmit an image in a valuable form. The first image in the stereo pair is called as reference image (First Left and then Right image also used as reference image after this work). Reference image is transformed into 2-D Discrete Cosine Transform (DCT II). The image divided into $8 \times 8$ block.

\subsection{Quantization}

This process performs the compression by dividing the DCT output of the pixel values of each block by a quantization coefficient then rounding down the result into the near integer. Quantization is carried out for the principle of making the image as more appealing by reducing the frequency through compression.

\subsection{Arithmetic Encoding}

Arithmetic encoding, a form of Entropy encoding used in lossless data compression is used in the proposed work. Any data compression approach, whether employing arithmetic coding, Huffman codes or any other coding technique, has a model which makes some assumptions about the data and the event encoded. The code itself can be independent of the model [4].

\subsection{Exhaustive Block Matching Algorithm}

In stereo image pair, first (left) image is used as reference image and its difference with the second image are estimated by Exhaustive Block Matching Algorithm. Disparity vector is the result of Block Matching Algorithm which is compressed into a bit stream arithmetic coding.

Disparity Estimation: Disparity estimation is fundamentally a correspondence problem. The correspondence between the two images can be determined by either matching features or small patches of gray values.

The displacement between the present block and the best matching block is the displacement vector.

\subsection{Decoding}

Decoding process of Left image as reference and right image as reference is shown in Fig 4 and Fig 5.

Stereo Image Decoding (Left image as reference image) To decode the image, all the steps in encoding were performed in reverse order.

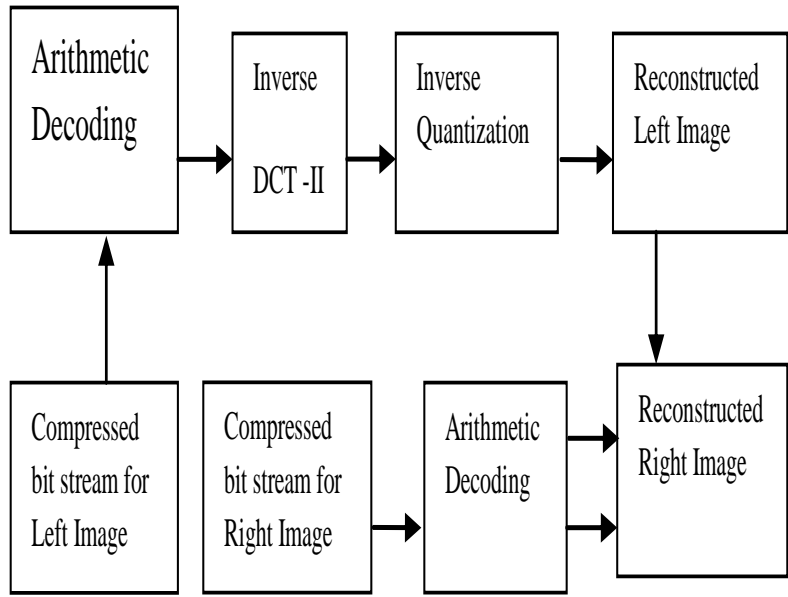

Fig 4: Flow of reconstructing the image

Stereo Image Decoding (Right image as reference image)

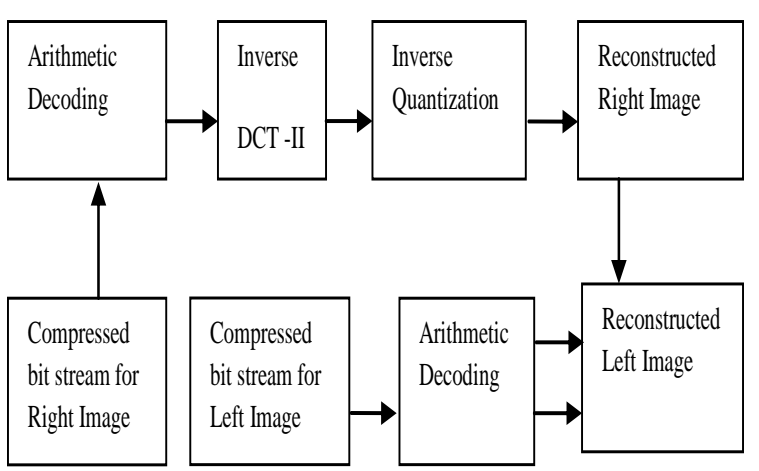

Fig 5: Flow of reconstructing the image 
Decoding performs the inverse process of quantization and 2D DCT II. Then it gets the reconstructed images which are decompressed images.

\section{RESULTS AND DISCUSSION}

\subsection{Test Images}

The experimental evaluation is performed using an assortment of images which are in .jpg format. The objects of images taken are: 1.Mouse box, 2.Wallet, 3.Stapler, 4. Two flowers and 5.Umbrella.

For experimentation purpose, images of five objects were selected. Fig 6 Shows that the images of stereo pair at 10, 30 and 50 degree. (a) (b) represents left and right images of stereo pair at 10 degree. Likewise (c) (d) represents images at 30 degree and (e) (f) represents images at 50 degree.

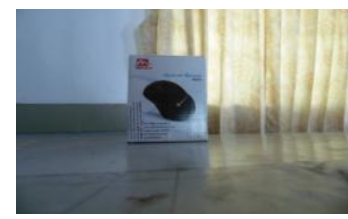

(a)

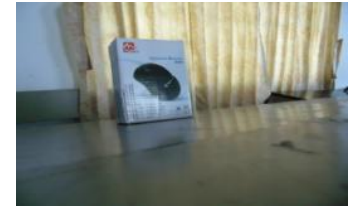

(c)

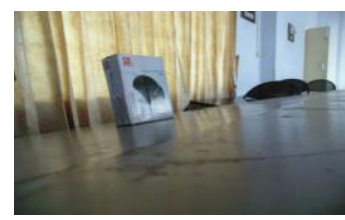

(e)

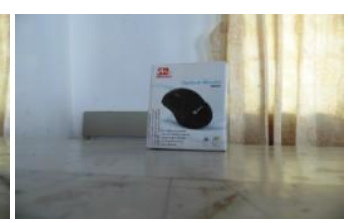

(b)

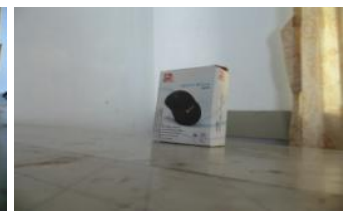

(d)

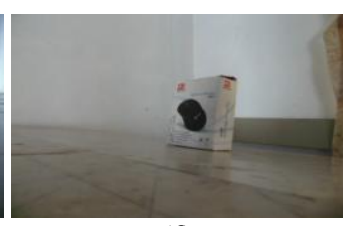

(f)
Fig 6: Left and Right images of stereo pair at 10,30 and 50 degree (Mouse box)
The proposed algorithm has been implemented in MATLAB and the results were obtained as tabulated in Tables 4.1, 4.2, $4.3,4.4,4.5$ and 4.6 .

\subsection{Compression and Decompression Time}

The compression time and decompression time is defined as the amount of time required to compress and decompress. Speed and complexity of the compressed image is shown by this parameter. The Compression Time for all the test images are shown in the Table 4.1. The Decompression Time for all the test images are shown in the Table 4.2.

Table 4.1 Compression Time (in seconds)

\begin{tabular}{|c|c|c|c|c|}
\hline Objects & $\begin{array}{l}\text { Reference } \\
\text { Image }\end{array}$ & 10 degr & 30 degr & $\begin{array}{l}50 \\
\text { degree }\end{array}$ \\
\hline Mouse Box & Left Image & 4.43 & 4.22 & 4.43 \\
\hline Mouse Box & Right Image & 4.10 & 4.12 & 3.65 \\
\hline Wallet & Left Image & 4.35 & 4.19 & 4.45 \\
\hline Wallet & Right Image & 4.27 & 4.08 & 3.87 \\
\hline Stapler & Left Image & 4.17 & 4.14 & 4.31 \\
\hline Stapler & Right Image & 3.95 & 3.85 & 3.63 \\
\hline Two Flowers & Left Image & 4.59 & 4.29 & 4.32 \\
\hline Two Flowers & Right Image & 4.36 & 4.14 & 3.90 \\
\hline Umbrella & Left Image & 4.09 & 4.28 & 4.56 \\
\hline Umbrella & Right Image & 4.22 & 3.97 & 3.78 \\
\hline
\end{tabular}

From the data in tables 4.1, it is observed that the compression time is lesser when the right image is used as reference image for all the angles 10, 30 and 50. It is also observed that with the increase of the angle between the images the compression time decreased, but at some angles compression time increased with the increase of angle of deviation.

The comparison chart for compression time is shown in the Fig.7.

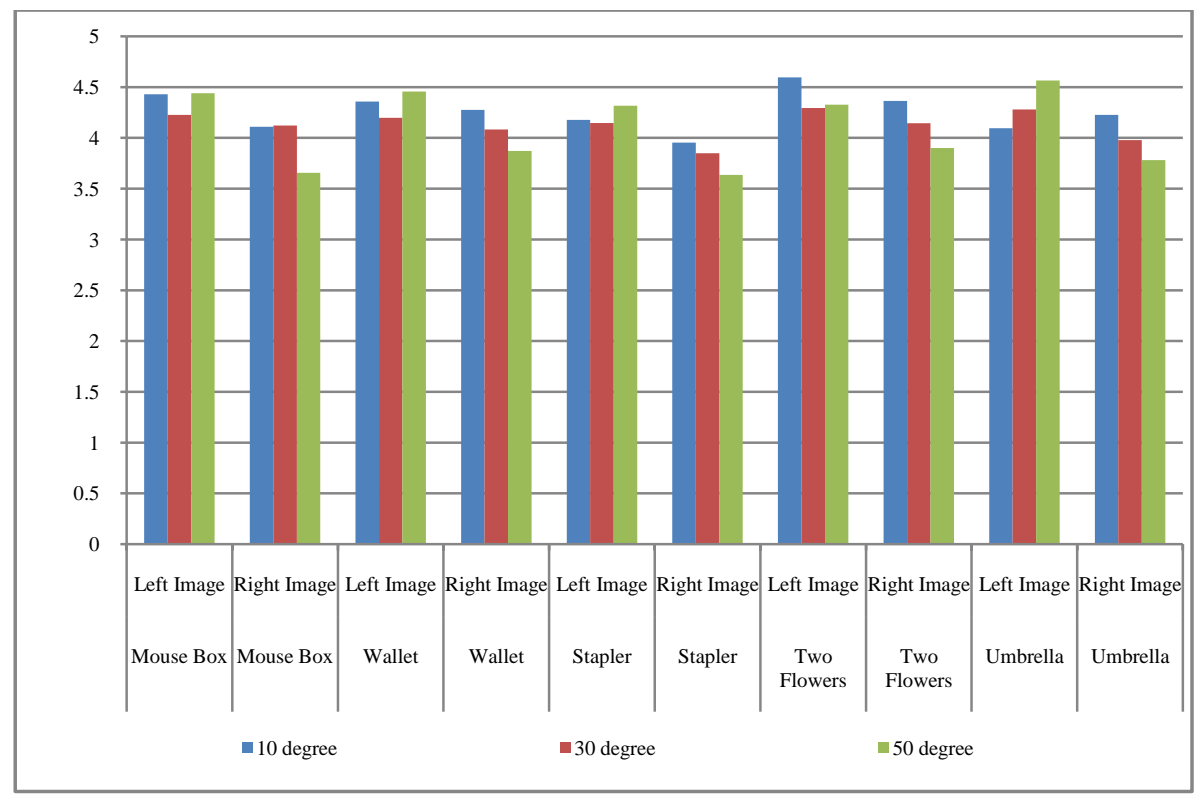

Fig 7: Comparison Chart for Compression Time 
From the data in tables 4.2 , it is observed that the Decompression time is lesser when the right image used as reference image for all the angles 1030 and 50. It is also observed that with the increase of the angle between the images the decompression time decreased, but at some angles decompression time increased with the increase of angle of deviation.

The comparison chart for Decompression time is shown in the Fig.8.

Table 4.2 Decompression Time (in seconds)

\begin{tabular}{|l|l|r|r|r|}
\hline Objects & Reference Ima & 10 degr & 30 degr & 50 degr \\
\hline Mouse Box & Left Image & 19.41 & 19.60 & 18.89 \\
\hline Mouse Box & Right Image & 19.44 & 19.59 & 17.79 \\
\hline Wallet & Left Image & 20.29 & 19.84 & 19.04 \\
\hline
\end{tabular}

\begin{tabular}{|l|l|r|r|r|}
\hline Wallet & Right Image & 19.71 & 19.04 & 17.79 \\
\hline Stapler & Left Image & 18.96 & 19.12 & 19.65 \\
\hline Stapler & Right Image & 18.82 & 18.18 & 17.84 \\
\hline Two Flowers & Left Image & 20.14 & 20.42 & 20.10 \\
\hline Two Flowers & Right Image & 19.27 & 19.88 & 18.31 \\
\hline Umbrella & Left Image & 19.41 & 19.71 & 19.78 \\
\hline Umbrella & Right Image & 19.52 & 18.95 & 18.14 \\
\hline
\end{tabular}

In Decompression time, mostly right image used as reference image results are better than left image used as reference image.

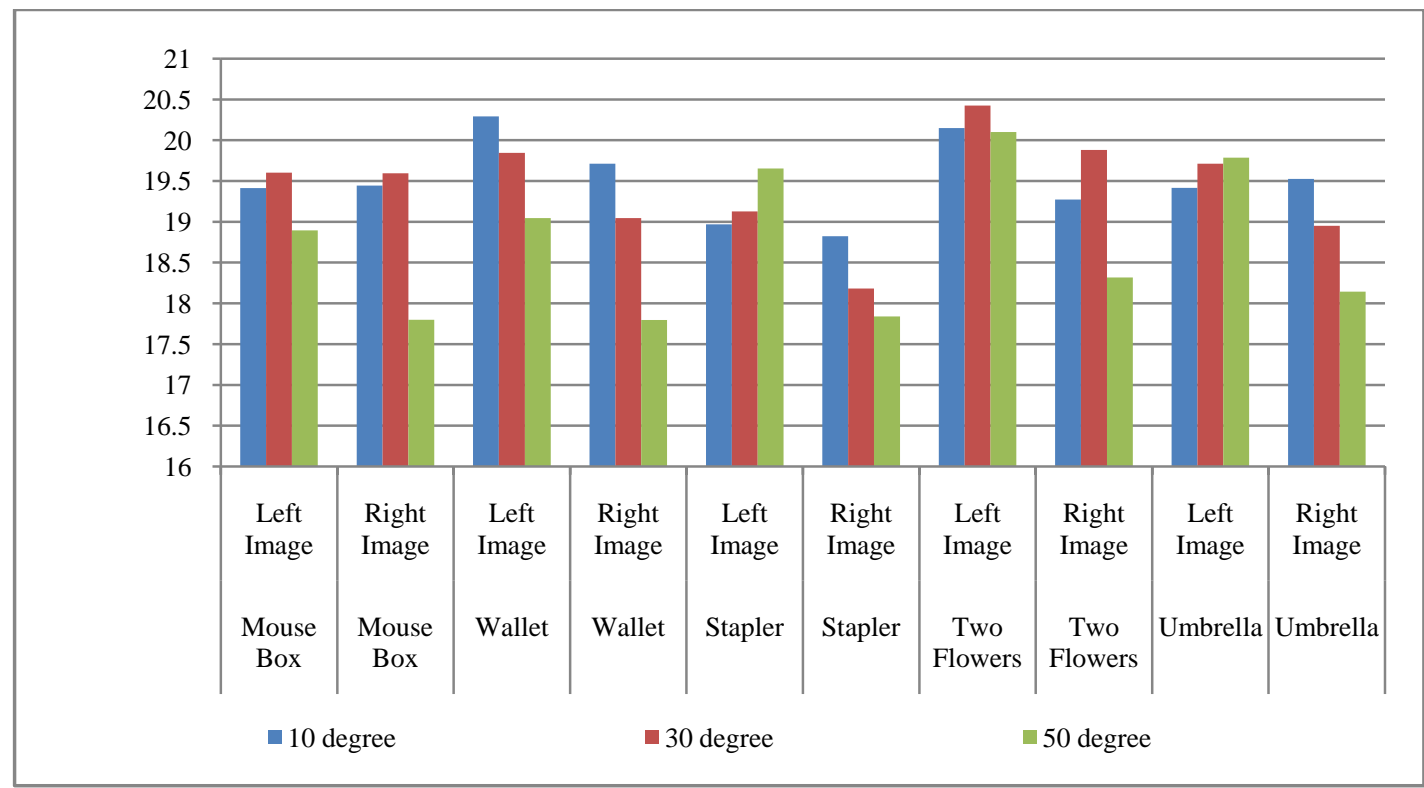

Fig 8: Comparison Chart for Decompression Time.

\subsection{Peak-Signal-To-Noise-Ratio}

The Peak Signal to Noise Ratio is used as a quality measurement between the original and the decompressed image. The higher PSNR indicates that the reconstruction image quality is higher. The PSNR values for the Left image used as reference image are shown in the Table 4.3. The comparison chart for PSNR (Left reference) is shown in Fig.9.
Table 4.3 PSNR (in dB) (Ref Left)

\begin{tabular}{|l|l|l|l|r|}
\hline Objects & Images & $\begin{array}{l}\mathbf{1 0} \\
\text { degree }\end{array}$ & 30 degr & 50 degr \\
\hline Mouse Box & Left Image & 48.10 & 49.96 & 53.95 \\
\hline Mouse Box & Right Image & 48.10 & 47.23 & 53.95 \\
\hline Wallet & Left Image & 47.05 & 49.01 & 51.54 \\
\hline Wallet & Right Image & 47.05 & 47.67 & 51.54 \\
\hline Stapler & Left Image & 48.42 & 49.37 & 53.29 \\
\hline Stapler & Right Image & 48.42 & 48.19 & 53.29 \\
\hline Two Flowers & Left Image & 41.42 & 42.02 & 44.35 \\
\hline Two Flowers & Right Image & 41.42 & 45.22 & 44.35 \\
\hline Umbrella & Left Image & 47.12 & 46.65 & 47.45 \\
\hline Umbrella & Right Image & 47.12 & 47.71 & 47.45 \\
\hline
\end{tabular}




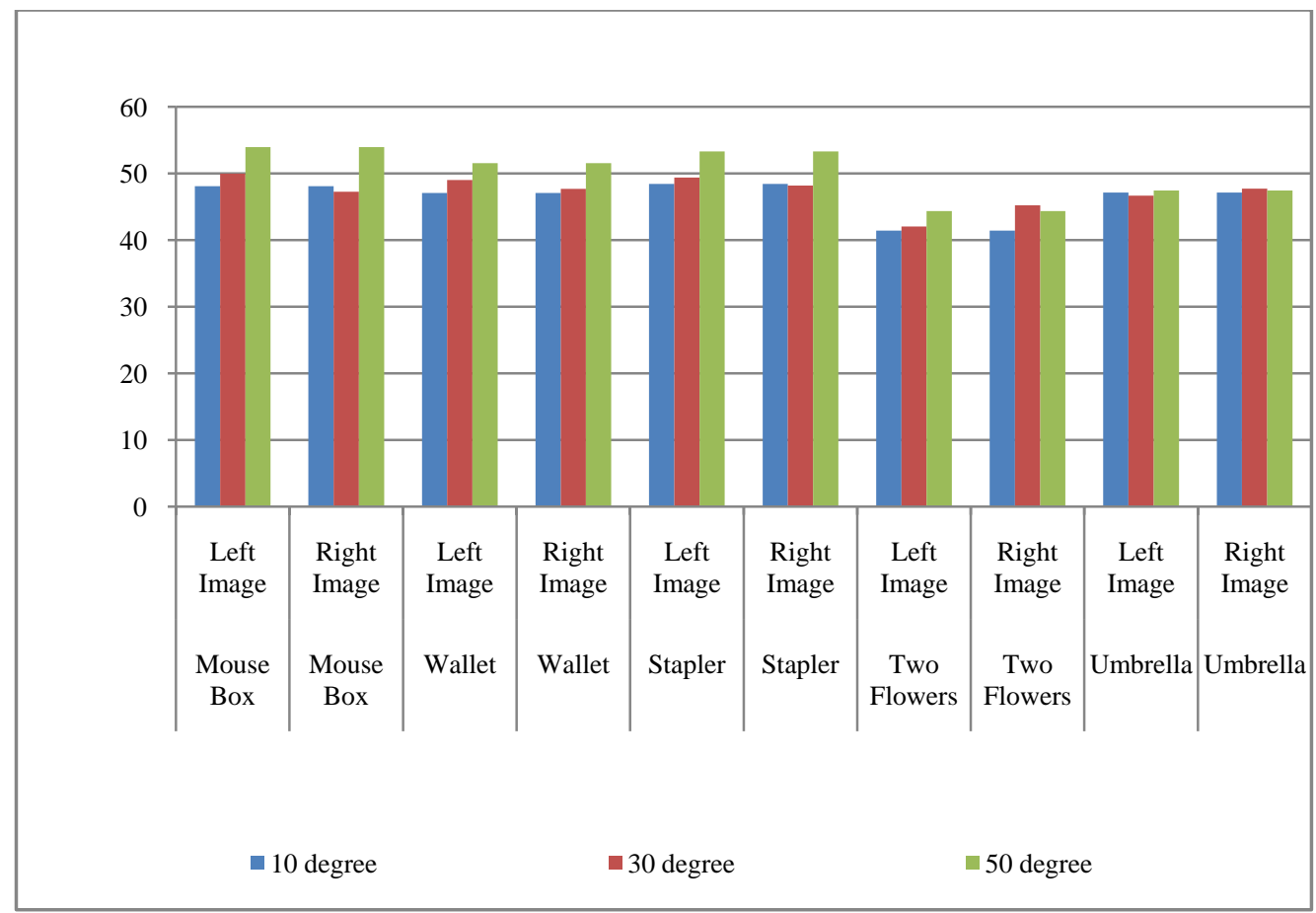

Fig 9: Comparison Chart for PSNR (Ref Left)

Based on the experimental results, PSNR values are better when left image is used as reference image than the right image. When the angle of deviation increases, the PSNR values also increase and provide good quality images.

The PSNR values for the Right image used as reference image are shown in the Table 4.4. The comparison chart for PSNR (Right reference) is shown in Fig.10.
Table 4.4 PSNR (Ref Right)

\begin{tabular}{|l|l|c|c|c|}
\hline Objects & Images & 10 degr & 30 degr & 50 degr \\
\hline Mouse Box & Left Image & 46.98 & 49.96 & 47.55 \\
\hline Mouse Box & Right Image & 46.98 & 47.23 & 47.55 \\
\hline Wallet & Left Image & 46.87 & 49.01 & 47.10 \\
\hline Wallet & Right Image & 46.87 & 47.67 & 47.10 \\
\hline Stapler & Left Image & 47.62 & 49.37 & 46.83 \\
\hline Stapler & Right Image & 47.62 & 48.19 & 46.83 \\
\hline Two Flowers & Left Image & 44.02 & 42.02 & 46.02 \\
\hline Two Flowers & Right Image & 44.02 & 45.22 & 46.02 \\
\hline Umbrella & Left Image & 46.67 & 46.65 & 46.76 \\
\hline Umbrella & Right Image & 46.67 & 47.71 & 46.76 \\
\hline
\end{tabular}

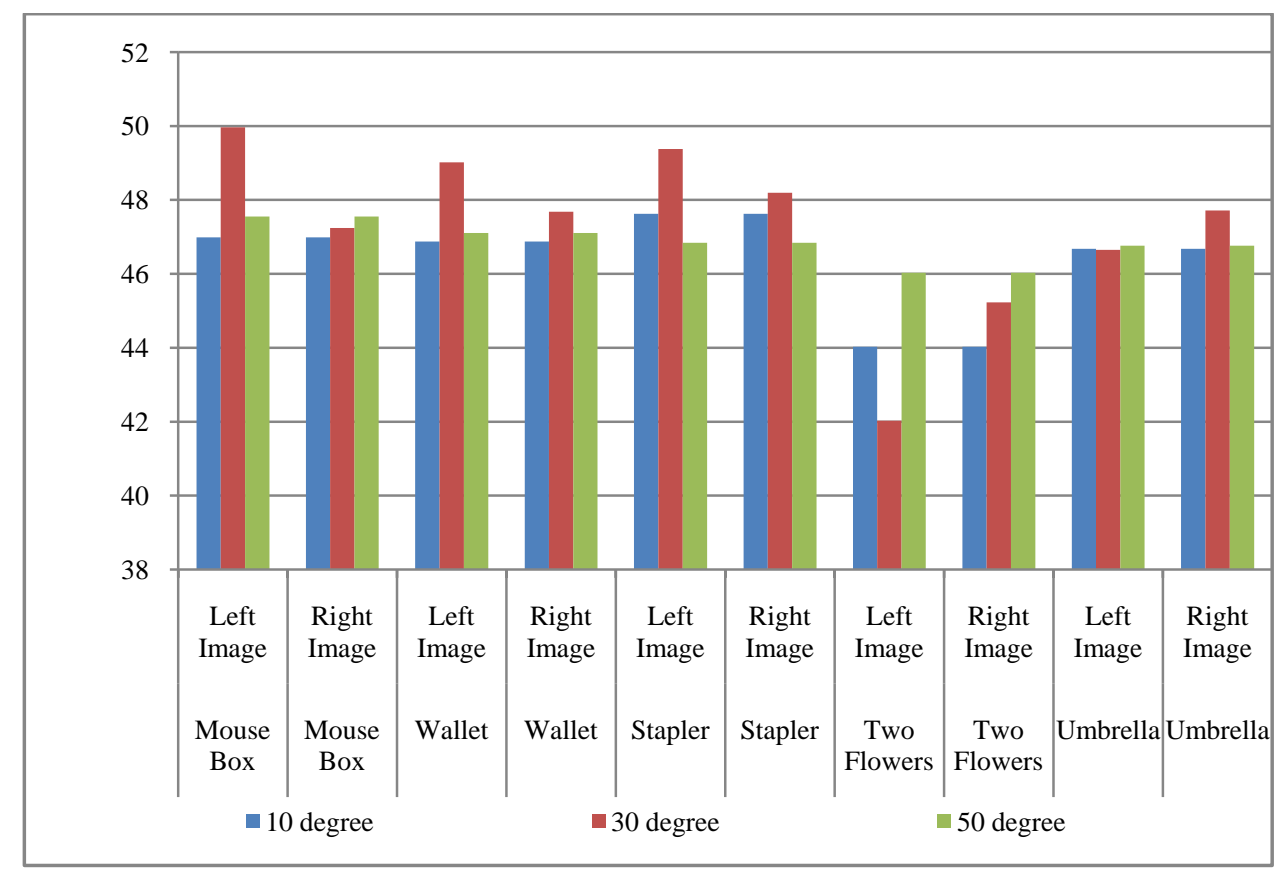

Fig 10: Comparison Chart for PSNR (Ref Right) 
PSNR values are better when left image is used as reference image than the right image and provide good quality images. The quality of image is very good, when the PSNR value goes high.

\subsection{Disparity and Bit Rate}

Each block in the first frame was compared with each block in the target frame. The block with the minimum error was rate values of images shown in Table 4.6. And comparison chart for bit rate shown in Fig.12. calculated. The disparity values of images shown in Table 4.5. And comparison chart for disparity shown in Fig.11. The bit

Table 4.5 Disparity

\begin{tabular}{|l|l|l|l|l|}
\hline Objects & $\begin{array}{l}\text { Reference } \\
\text { Image }\end{array}$ & $\begin{array}{l}\mathbf{1 0} \\
\text { degree }\end{array}$ & $\begin{array}{l}\mathbf{3 0} \\
\text { degree }\end{array}$ & $\begin{array}{l}\mathbf{5 0} \\
\text { Degree }\end{array}$ \\
\hline Mouse Box & Left Image & 304.97 & 305.04 & 306.20 \\
\hline Mouse Box & Right Image & 305.16 & 303.41 & 302.85 \\
\hline Wallet & Left Image & 304.13 & 304.90 & 306.03 \\
\hline Wallet & Right Image & 305.07 & 303.67 & 303.00 \\
\hline Stapler & Left Image & 304.62 & 305.19 & 306.35 \\
\hline Stapler & Right Image & 302.97 & 304.72 & 302.64 \\
\hline Two Flowers & Left Image & 304.16 & 305.18 & 305.50 \\
\hline Two Flowers & Right Image & 304.23 & 305.46 & 301.78 \\
\hline Umbrella & Left Image & 303.73 & 303.90 & 305.66 \\
\hline Umbrella & Right Image & 303.67 & 304.48 & 302.37 \\
\hline
\end{tabular}

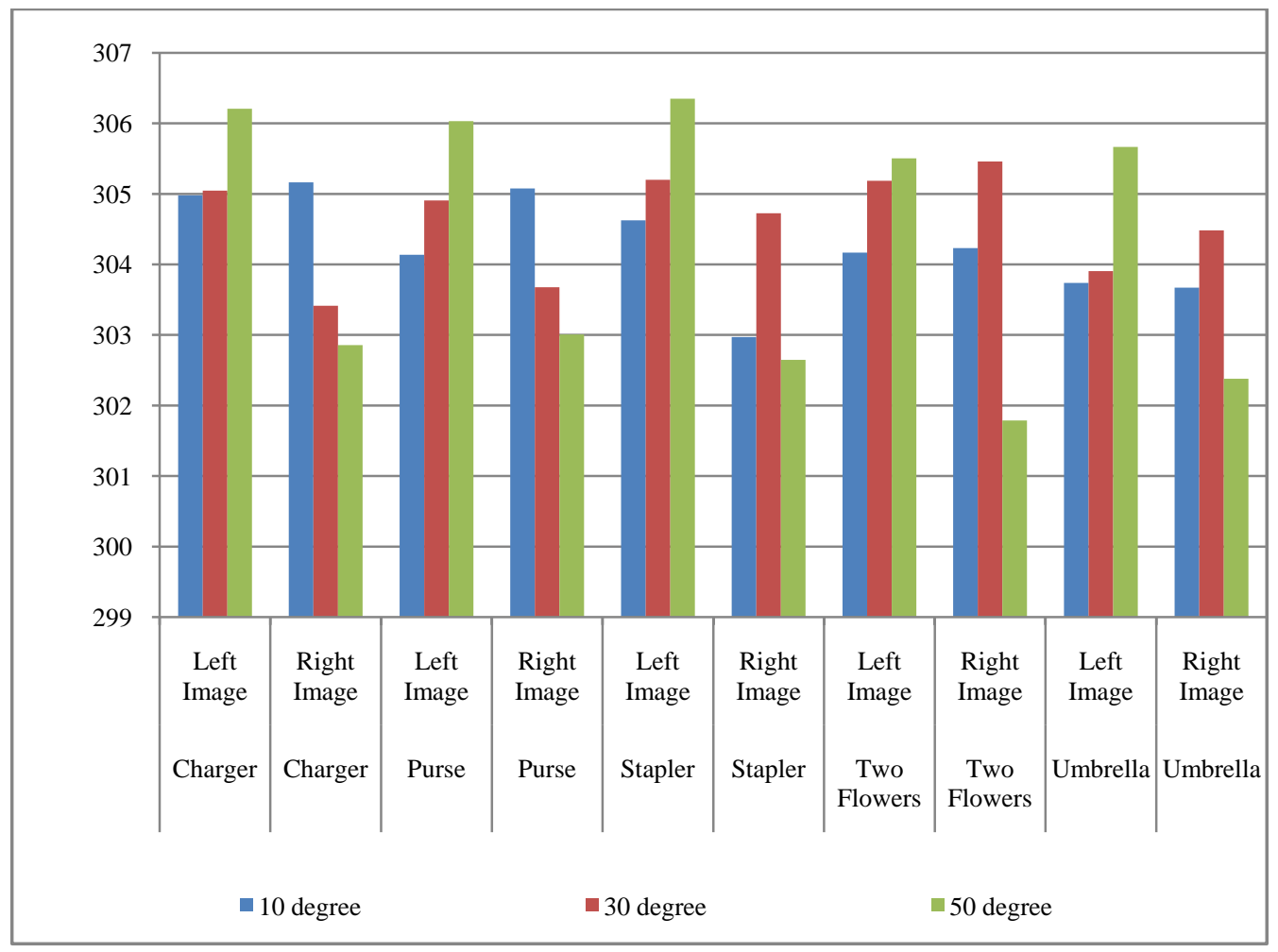

Fig 11: Comparison Chart for Disparity

Disparity values results are better when right images are used as reference image compared to left images. In some points when the angle of deviation were increased then disparity value was increased.

\section{Table 4.6 Bit rate}

\begin{tabular}{|l|l|r|r|r|}
\hline Objects & $\begin{array}{l}\text { Reference } \\
\text { Image }\end{array}$ & $\begin{array}{l}\text { 10 } \\
\text { degree }\end{array}$ & $\begin{array}{l}\text { 30 } \\
\text { Degree }\end{array}$ & $\begin{array}{l}\text { 50 } \\
\text { degree }\end{array}$ \\
\hline Mouse Box & Left Image & 1.639 & 2.0261 & 0.7063 \\
\hline Mouse Box & Right Image & 1.639 & 2.0261 & 0.7063 \\
\hline
\end{tabular}

\begin{tabular}{|l|l|r|r|r|}
\hline Wallet & Left Image & 1.3781 & 1.8395 & 0.9446 \\
\hline Wallet & Right Image & 1.3781 & 1.8395 & 0.9446 \\
\hline Stapler & Left Image & 1.8932 & 2.2286 & 0.6728 \\
\hline Stapler & Right Image & 1.8932 & 2.2287 & 0.6728 \\
\hline Two Flowers & Left Image & 1.1066 & 1.702 & 0.6106 \\
\hline Two Flowers & Right Image & 1.1066 & 1.7019 & 0.6106 \\
\hline Umbrella & Left Image & 1.6456 & 2.1087 & 0.4247 \\
\hline Umbrella & Right Image & 1.6456 & 2.1087 & 0.4247 \\
\hline
\end{tabular}




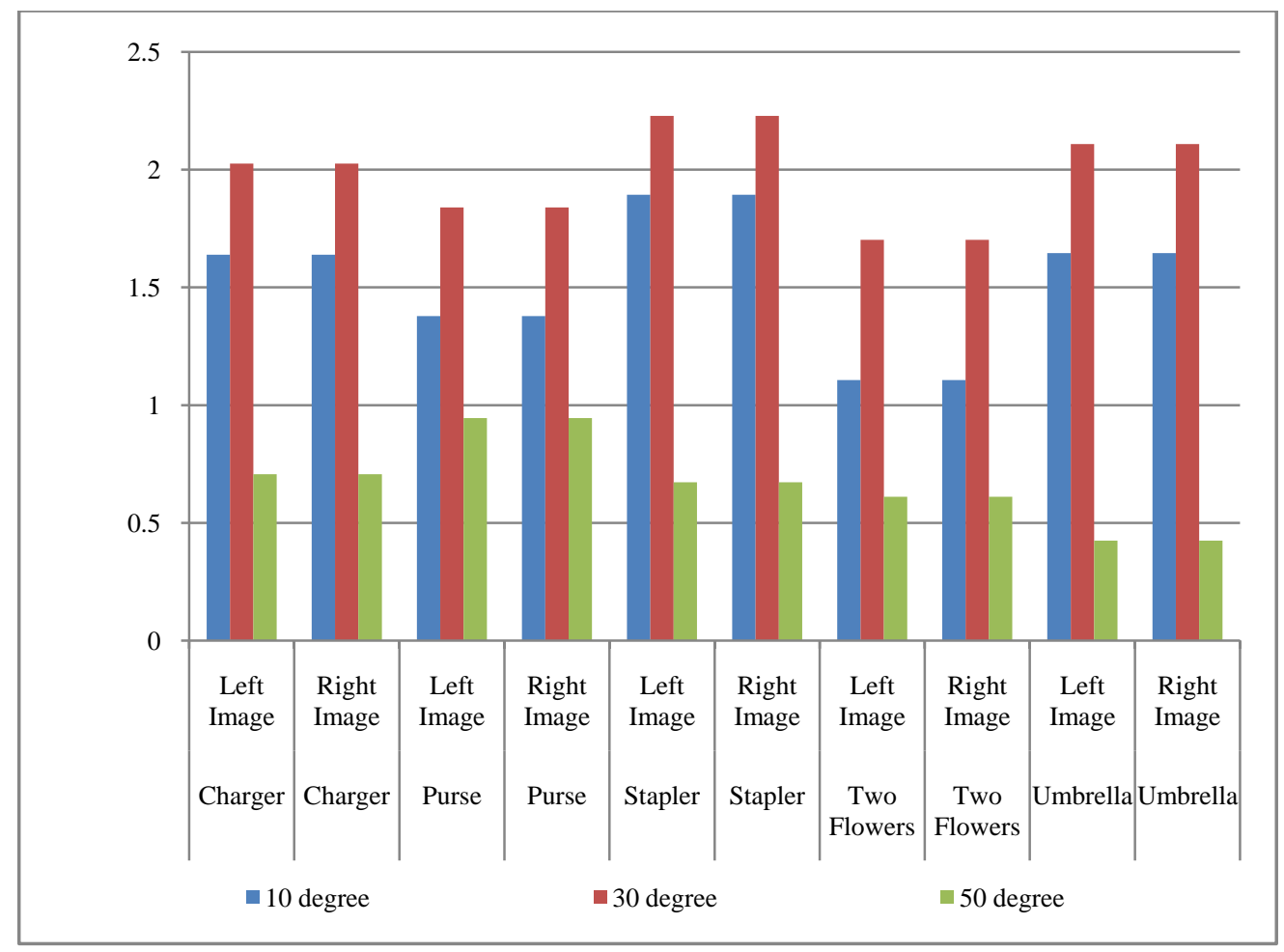

Fig 12: Comparison Chart for Bit rate.

Compression efficiency is measured by the bit rate. Bit rate values remain same for both left images and right images when used as reference image.

\section{CONCLUSION AND FUTURE ENHANCEMENT}

In this proposed work, right image along with the left image is used as reference image and real time stereo images are used for compression. The images are taken in different angles between 10, 30 and 50 degree. All the images are in .jpg format. The quality parameters like Compression Time, Decompression Time, PSNR, Disparity and Bit rates are calculated for all the two set stereo images of five objects. The results of Compression Time and Decompression Time have shown better results than right image is used as reference image but the image quality is better when the left image is used as reference image. Disparity values results are better when right images are used as reference image compared to left images. Bit rate values are same with both the references.

In future, the quality of stereo image compression may be compared by applying different block matching algorithms

\section{REFERENCES}

[1] A.Loussert, A.Alfalow, R.Elsawda and A.Alkholidi, "Enhanced Systems for Images Compression and Encryption by addition of Biometric Characteristics", International Journal of Software Engineering and its Application, Vol.2, No.2, April 2008, PP 111-118.
[2] Anil.K.Jain, "Fundamental of Digital Image Processing", PHI, 1991.

[3] Ashish Agarwal, "Compressing Stereo Images Using a Reference Image and the Exhaustive Block Matching Algorithm to Estimate Disparity between the Two Images", International Journal of Advanced Science and Technology, Vol. 32, July, 2011, PP 1-12.

[4] D.Chandradevi and M.Sundaresan, "Exhaustive Block Matching Algorithm to Estimate Disparity between Stereo Images“, (ISSN -7529; ISBN 978-93-80544-20-5) 10th INDIACom; INDIACom-2016; IEEE Conference ID: 374652016 3rd International Conference on Computing for Sustainable Global Development, 16-18th March, 2016, PP 4898-4903.

[5] Marco Zamarin, "Loseless Compression of Stereo Disparity Maps for 3D", IEEE International Conference on Multimedia and Expo Workshops, 2012, PP 617-622.

[6] T.Ramaprabha and Dr.M.Mohamad Sathik, "Analysis of Stereo Image Pair Compression Non Immersive Virtual Reality World using DCT II and BTC", International Journal on Advanced and Innovative Research, December 2012, PP 589-593.

[7] Tamas Frajka, Kenneth Teger, "Residual Image Coding for Stereo Image Compression", Optical Engineering, Vol.42, No.1, January 2003, PP 1-8. 